\title{
Design of hydrophobic surfaces for liquid droplet control
}

\author{
Akira Nakajima* \\ Tokyo Institute of Technology, Japan
}

\begin{abstract}
Technologies related to hydrophobic coatings have been applied to various industrial items for droplet formation and removal from solid surfaces. However, for such surfaces, excellent water-shedding properties and droplet control in a desired direction are not easily attainable merely by reducing their surface energies. Success requires the design and control of a nanolevel surface structure with appropriate chemical composition. Various hydrophobic surfaces have been developed to date, and their static hydrophobicity has been investigated carefully. Moreover, studies of dynamic hydrophobicity of droplets have increased recently because of the rapid advance and availability of high-speed cameras, facilitating direct evaluation of droplet motion. This paper presents a review of recent studies investigating the design of hydrophobic surfaces for liquid droplet control, specifically focussing on recent studies of dynamic hydrophobicity.
\end{abstract}

D uring rainfall, small water droplets can be observed to roll off hydrophobic umbrella surfaces at different speeds. A droplet on a round hydrophobic surface does not always slide down on the surface easily at a small tilt angle, but even if it does, large moving acceleration is not always observed. What determines the motion of droplets on solid surfaces?

Differences in droplet shape on solid surfaces are often explained by variations in wettability. The wetting of a solid surface has persisted through the ages as a research subject at the border between physics and chemistry. Wettability control of a solid against a liquid has been widely explored in industry for application in daily life because it can engender various physical and chemical phenomena related to solids and liquids. Wettability is an important property of solid surfaces from both fundamental and practical aspects. The degree of adhesion of liquid droplets onto a solid surface is commonly described in terms of the contact angle $(\theta)$, which is given by Young's equation as

$$
\gamma_{\mathrm{sv}}=\gamma_{\mathrm{sl}}+\gamma_{\mathrm{lv}} \cos \theta
$$

where $\gamma_{s v}, \gamma_{\mathrm{s}}$, and $\gamma_{\mathrm{lv}}$ represent the interfacial free energies per unit area of the solid-gas, solid-liquid and liquid-gas interfaces.

Although the definition and measurement of the contact angle are simple and easy, the nature of wettability is complex because it is related to three phases: solid, liquid and gas. Moreover, various factors affect the surface wettability of a solid. When one factor of a solid surface such as roughness is altered, other factors such as the surface topography or its chemical composition often change simultaneously. Therefore, it is usually difficult to discuss the results of surface wettability obtained from experiments while retaining academic rigor. The main research interest for industrial materials is the behavior of millimeter-size droplets on a solid surface of more than a few centimeters in area. Therefore, the discussion of wettability among samples with great differences in surface character is sometimes conducted with ignorance of the influence of other characteristics.

Hydrophobic coatings are extremely important for wettability control. The coatings are anticipated for various industrial uses such as anti-wetting, anti-snow (or ice), anti-rust and reduced friction resistance by reducing solid-liquid interaction. Generally, a hydrophobic surface is one on which water forms a round droplet that is easily removed. Recent advances in probe microscopy and surface spectroscopy and the wider availability of such techniques have revealed that macroscopic surface hydrophobicity, especially dynamic hydrophobicity, is affected by nanolevel characteristics such as structure, chemical composition and their alignment and homogeneity. The expected properties for a hydrophobic surface cannot always be obtained unless precise design and control of the solid surface are applied. This report presents the results of recent studies on the design of hydrophobic surfaces for droplet removal or control from the viewpoint of materials science.

\section{Surface factors for hydrophobicity}

As described above, the contact angle is determined by the balance among interface energies. The wettability of a solid surface by a liquid decreases concomitantly with increasing contact angle. Based on the theories of Dupre and Girifalco-Good in their work on adhesion [1], the contact angle can be described as follows.

$$
\cos \theta=2 \phi \sqrt{\gamma_{\mathrm{sv}} / \gamma_{\mathrm{lv}}}-1
$$

where $\phi$ is a correction factor. Relations between solids and liquids typically have a value of $\phi$ that approaches unity. This equation implies that a decrease in $\gamma_{\mathrm{sv}}$ is necessary for an increase in contact angle. By assuming $\phi$ and the surface energy of water to be unity and $72.8 \mathrm{~mJ} \mathrm{~m}^{-2}$, respectively, the maximum contact angle on a flat surface that is attainable merely by lowering the surface energy (the lowest surface energy value yet recorded is ca. $6 \mathrm{~mJ} \mathrm{~m}^{-2}$ [2], for a surface with regularly aligned closest-hexagonal-packed $\mathrm{CF}_{3}$ group) is calculable as ca. $115.2^{\circ}$.

When roughness is imparted to a solid surface, its wettability is changed. Wenzel [3] modified Young's equation and described the contact angle $\theta^{\prime}$ on a rough surface as follows:

$$
\cos \theta^{\prime}=r\left(\gamma_{\mathrm{sv}}-\gamma_{\mathrm{sv}}\right) / \gamma_{\mathrm{sv}}=r \cos \theta
$$

In these equations, $\theta$ denotes the contact angle on a smooth surface. In addition, $r$ signifies the roughness factor, which is defined as the ratio 
of the actual area of a rough surface to the geometrically projected area. This $r$ differs from the value of the arithmetic mean deviation of the profile $(R a)$, which is commonly employed in the engineering field. In this equation, $r$ is always greater than unity. Therefore, surface roughness enhances the hydrophobicity of a hydrophobic surface.

Cassie proposed an equation for a hydrophobic surface with large roughness. With increasing surface roughness, air intrudes into the hydrophobic solid-liquid interface. It is assumed that the interface comprises solid and air. When the unit area of the surface has a wetted solid surface area fraction $f$ with a water contact angle $\theta$, the contact angle on the surface can be expressed as follows assuming a $180^{\circ}$ water contact angle for air [4]:

$$
\cos \theta^{\prime}=f \cos \theta+(1-f) \cos 180^{\circ}=f \cos \theta+f-1
$$

Figure 1 presents the models of Wenzel and Cassie. When the surface roughness is small, Wenzel's mode is dominant. However, Cassie's mode becomes dominant with increasing surface roughness. Johnson [5] simulated the contact angle of a water droplet on idealized sinusoidal surfaces, demonstrating that the dominant mode transition from Wenzel to Cassie occurs when the roughness factor becomes greater than a certain level (ca. 1.7). In a practical hydrophobic surface with roughness, both modes will exist. An excellent water-shedding property on a superhydrophobic surface (described later) is attained by increasing the contribution of Cassie's mode, which reduces the practical contact area between the solid and liquid [6]. McHale et al. [7] theoretically examined the relationship between perturbations to contact angles on a rough or textured surface and the superhydrophobic enhancement of the equilibrium contact angle. They derived the idea that a contact angle increase on a rough surface is accompanied by amplification for perturbations to the contact angle in the Wenzel case and by an attenuation for perturbations in the Cassie case. Their results predict that a Wenzel surface will become 'sticky', whereas a Cassie surface will become 'slippery'.

In the Wenzel and Cassie equations, there are two important factors for surface wettability: both the alignment of chemical composition and roughness and the roughness shape are ignored. The compositional difference inside the three-phase contact line provides no remarkable difference in the contact angle [8]. The contribution of Cassie's mode to overall hydrophobicity is commonly increased when the surface has a large area into which water has difficulty penetrating (such as the bottom edge of a rectangular hydrophobic channel) [9]. The hydrophobicity of the solid is enhanced by surface roughness. However, the degree depends on the surface shape. Therefore, an additional function can be provided to the hydrophobic surface by designing a roughness shape (Figure 2) [10]. To date, various limitations and corrections have been pointed out and proposed for these two models [7-9,11-17]. Drelich et al. [11] modified the Cassie equation to include a term representing the line tension contribution to the three-phase system. Woodward et al. [12] systematically measured contact angles on chemically heterogeneous surfaces where the length scale of the heterogeneity was $10-100 \mathrm{~nm}$. They reported the contribution of the boundary amount and the length scale of surface heterogeneity to overall hydrophobicity. Patankar [13] proposed a design model to develop a superhydrophobic substrate that accounts for multiple equilibrium drop shapes. Long et al. [15] proposed thermodynamic models for contact angles on rough, heterogeneous surfaces using a parameter named the 'surface feature factor' to classify surfaces into three categories: roughness-dominated, heterogeneity-dominated, and mixed-rough heterogeneous. Marmur et al. [17] pointed out that the Wenzel and Cassie equations are not expected to be valid when the droplet size approaches the order of magnitude of the wavelength of roughness or chemical heterogeneity, and that both the local equilibrium condition at the contact line and the global equilibrium condition involving the wetted area within the contact line are necessary and complementary. However, as a first approximation, Wenzel's and Cassie's equations are applicable to the relationship between solid surface structure and wettability in many cases.

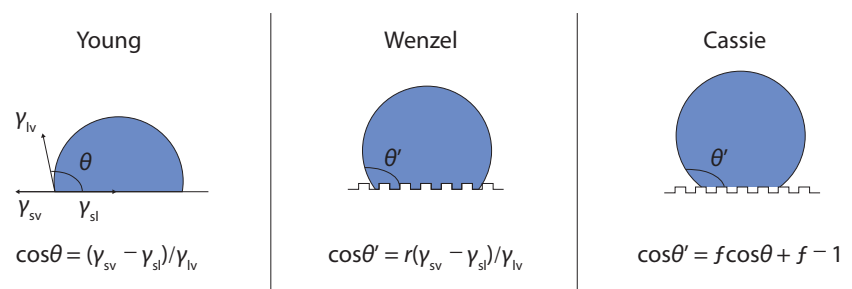

Figure 1. Young, Wenzel, and Cassie models for hydrophobicity of solid surfaces with and without roughness
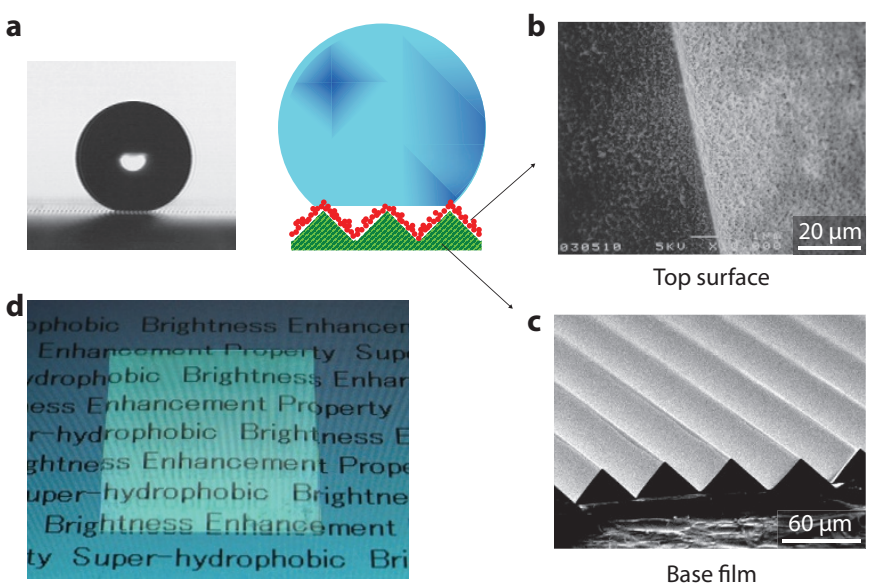

Figure 2. Transparent superhydrophobic polymer films with brightness enhancement for backlighting. The surface shape is prepared using brightness-enhancement film (BEF), fluoroacrylic resin and colloidal silica. (a) Droplet shape on the transparent superhydrophobic film prepared from BEF with indium tin oxide (ITO) top coating, (b) scanning electron micrograph of the same film, and (c) scanning electron micrograph of a BEF. Adapted from K. Takeda et al., J. Mater. Sci. Lett. 20, 2131 (2001) (๔ 2001 Springer). (d) Brightness enhancement effect in a superhydrophobic film prepared from a BEF with ITO (tilt: $30^{\circ}$ ).

Moreover, there are differences in the degree of contribution of the alignment of roughness or chemical composition in the hydrophobic surfaces to sliding angle and dynamic sliding behaviors such as sliding acceleration (described later). Therefore, precise control of the surface composition and its structure is necessary to attain static and dynamic hydrophobicity simultaneously. With proper design of the surface structure and chemical composition, control of directional transportation and sliding of a liquid droplet becomes possible.

\section{Superhydrophobic surfaces}

Surfaces, materials and states having water contact angles greater than $150^{\circ}$ are generally designated as superhydrophobic. As described in the preceding section, such surfaces are not attainable merely by reducing the surface energy. All superhydrophobic surfaces and coatings have been obtained by combining surface roughness with lower surface energy [18-21]. Recently, several examples of highly hydrophobic (contact angle $>120^{\circ}$ ) surfaces comprised solely of inorganic materials with intrinsic surface energy higher than that of organic compounds were reported [22,23]. These results are expected to be related to the specific surface shape and high adsorption capability of organic compounds.

Quéré et al. [24] sandwiched a water droplet between two superhydrophobic plates under loading to demonstrate that the water contact angle decreases drastically after de-loading. This result implies that Cassie's mode appears as a metastable state even when Wenzel's mode is the most stable. These two wettability modes do not always appear as the most stable state. A similar wetting transition from Cassie to Wenzel has also been reported based on experiments involving 
a

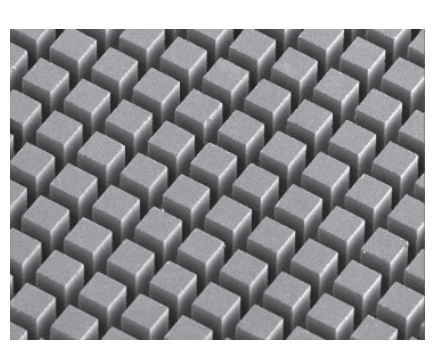

b

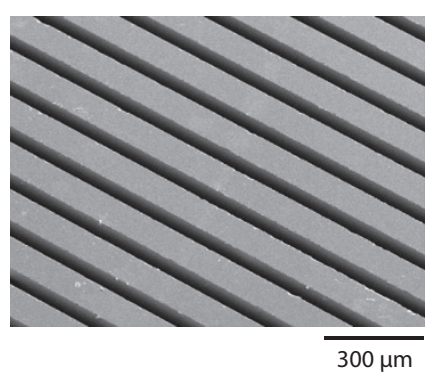

C

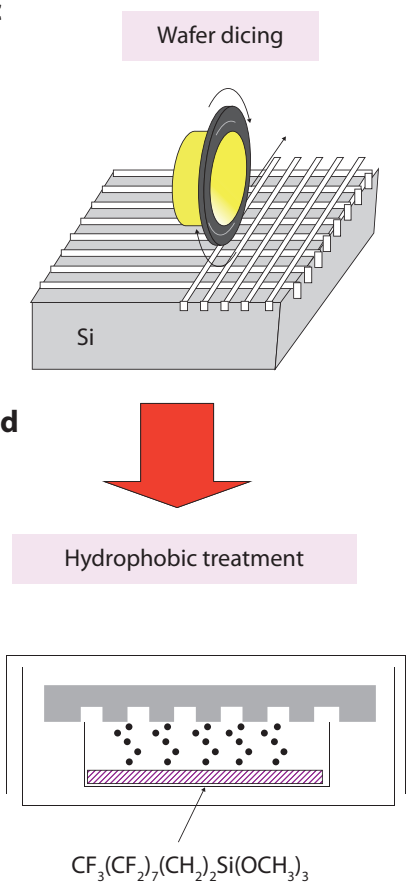

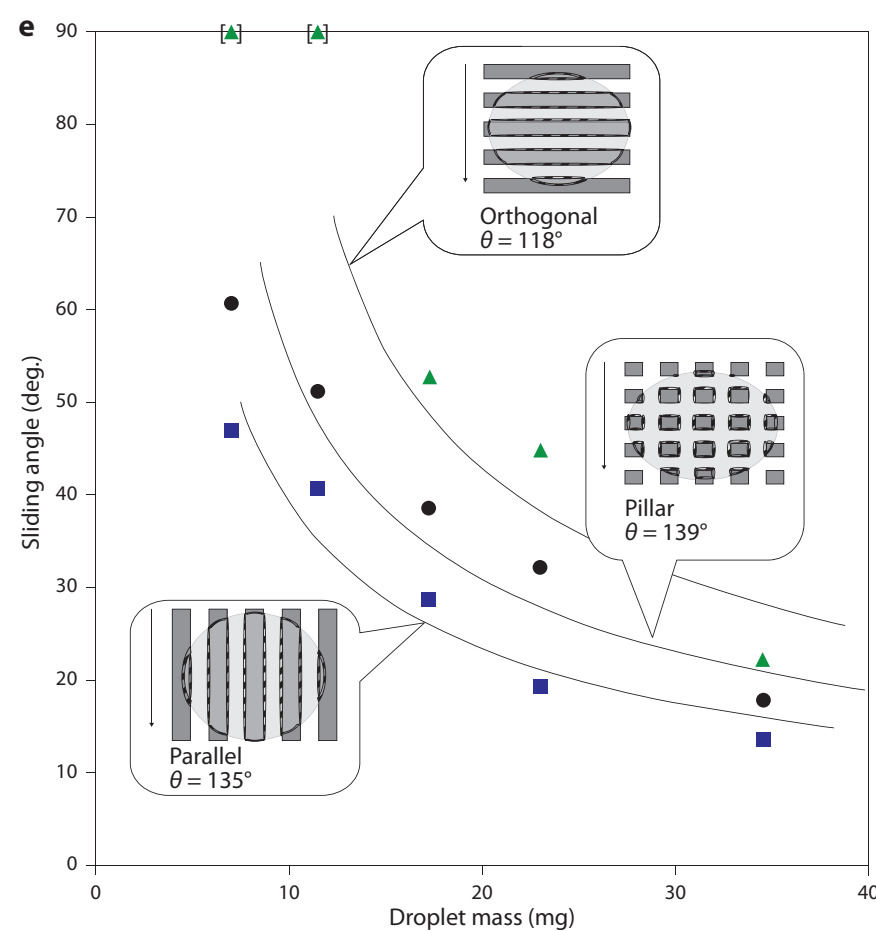

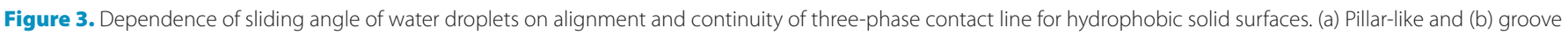

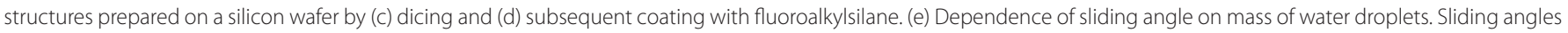



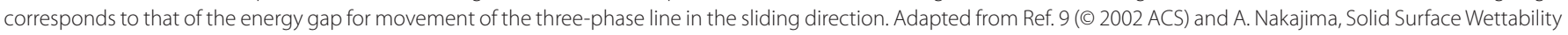
and its Control (in Japanese) (๔ 2007 Uchida Rokakuho Publishing, Tokyo, Japan).

evaporation [25], condensation [26,27] and vibration [28]. This transition is accompanied by a decrease in the contact angle or an increase in contact angle hysteresis [24,26]. The stability of these two modes is discussed with consideration of the surface structure and energy balance [29-31]. Barbieri et al. [29] examined the wetting mode transition of a surface comprised of flat-topped pillars from theoretical and experimental perspectives, reporting that the transition was a function of pillar parameters determined geometrically from the surface roughness. Ishino et al. [30] presented energy state diagrams in the twodimensional space of texture parameters, which suggests transitions between the wetting states. They proposed a model that allows the description of transition states between (meta)stable contact states and which facilitates quantification of the energy barriers between them. Patankar [55] proposed a methodology, based on energy balance, to determine whether a transition from the Cassie to Wenzel case is possible. Results showed that the more stable configuration is always that with the smallest contact angle.

Superhydrophobic surfaces do not maintain a state once the energy balance is broken by some means. Therefore, this state is attainable under a specific condition and sustained under small external field fluctuation. Both the contact angle and sliding angle of the superhydrophobic state depend on the relationship between droplet size and roughness level. For small droplets, in many cases the surface roughness does not enhance hydrophobicity because the droplet does not reflect the surface area increase realized by imparting surface roughness. However, when a droplet is large compared to the surface roughness, the droplet subducts into the roughness, and the contribution of Wenzel's mode increases. The sliding angle under this state depends on the droplet mass and the resistance to droplet sliding due to surface roughness.

Recently, many studies of superhydrophobic surfaces have been conducted worldwide. The contents extend from a new processing route for surfaces to droplet behavior on the surface, including sliding [6,9,32-34], freezing [35-37], evaporation [25,38-40] and bouncing [41-43]. However, such surfaces have not been put into practical use except in very limited cases. Superhydrophobic surfaces require surface roughness. Therefore, long-time outdoor durability and mechanical strength are difficult to obtain. Surface roughness engenders a place for stain accumulation on the surface; surfaces with high roughness commonly show poorer abrasion resistance than flat surfaces [44]. Especially in the case of transparent superhydrophobic surfaces, surface roughness is expected to be less than $100 \mathrm{~nm}(R \mathrm{a})$ in order to suppress visible light scattering. Such fine structures are easily broken by pressure or abrasion. Although the introduction of $\mathrm{a} \mathrm{TiO}_{2}$ photocatalyst [45], a roughness mixture [46] and conductivity [10] has been attempted in order to improve the durability of superhydrophobic surfaces against outdoor exposure, the effects of these approaches differ greatly from those required for practical use. Natural lotus leaves possess self-renewal capability; clean wax is supplied to the surface by their own metabolism. These two functions maintain the superhydrophobicity of the plant surface for its entire lifetime. Unfortunately, studies to overcome these weak points for artificial superhydrophobic surfaces are not often reported. To make superhydrophobic surfaces a key technology in the field of surface functional materials, the development of technologies for the improvement of mechanical strength and long-term outdoor durability is indispensable. Otherwise, a superhydrophobic surface is merely an object to satisfy the curiosity of materials chemists. Breakthrough studies are necessary in this area.

\section{Dynamic hydrophobicity}

Furmidge [47] derived an equation that describes the relationship between the sliding angle and the receding and advancing contact angles as follows:

$$
\frac{m g \sin \alpha}{w}=\gamma_{\mathrm{vl}}\left(\cos \theta_{\mathrm{R}}-\cos \theta_{\mathrm{A}}\right)
$$

where $\alpha$ denotes the sliding angle, $m$ is the mass of the water droplet, $g$ is gravitational acceleration, $w$ is droplet width and $\theta_{\mathrm{R}}$ and $\theta_{\mathrm{A}}$ are the 
receding and advancing contact angles. The angles $\theta_{\mathrm{R}}$ and $\theta_{\mathrm{A}}$ are the critical angles at which a droplet can stay on a surface without sliding on an inclined surface by its own shape deformation. Therefore, when the difference of these angles is small, the sliding angle of the droplet is small. Although modified relations between the sliding angle and the receding and advancing contact angles have been proposed by Dussan and Carre $[48,49]$, the fundamental relationship between contact angle hysteresis and sliding angle is the same. Removal of the origins of contact angle hysteresis is an important approach for the design of surfaces with a small sliding angle. Chen et al. [50] proposed the following origins for contact angle hysteresis from the adhesion behavior of droplets on a mica surface coated with surfactants: (i) increasing the freedom of the surface molecules to reorder, (ii) increasing the load and time allowed for surfaces to remain in contact, and (iii) increasing the rate of separation or retraction. However, the respective and combined contributions of these factors to practical contact angle hysteresis remain unclear. Moreover, their contribution is expected to differ among materials.

Along with the contact angle, surface roughness and surface chemical composition (molecule length [51]) affect the sliding angle and the contact angle hysteresis. For a smooth hydrophobic surface, a surface with low surface energy provides a small sliding angle. However, for fluorocarbon-based hydrophobic surfaces, the sliding angle is commonly large despite a large contact angle [52-54]. Unlike the contact angle, the sliding angle is strongly affected by the alignment and continuity of the three-phase contact line $[9,32,55]$. It is feasible to lower the sliding angle on the surface with a small contact angle by appropriate design of the three-phase contact line (Figure 3). Structural heterogeneity and chemical heterogeneity in the hydrophobic surface affect the sliding angles more remarkably than the contact angles, increasing sliding angles while pinning the three-phase contact lines [56].

Although the sliding angle relates the motion or deformation of droplets, it is not an index of dynamic hydrophobicity, such as sliding acceleration or velocity. The sliding angle is not a function of time; its meaning is the limitation of interface energy balance at the three-phase contact line. Both the contact angle and sliding angle are essentially thermodynamic properties. The surface shape of industrial items is generally determined by their size, function and design. For the design of surfaces with excellent water-shedding performance, information about how fast the droplet can be removed from the surface at a certain tilt angle is becoming more important than information regarding the lowest tilt angle at which the droplet begins to slide. Moreover, dynamic hydrophobicity relates directly to energy saving and environmentally friendly technologies by decreasing the friction drag between liquids and solids. However, a fundamental understanding of the factors (hydrophobic solid surface characteristics) contributing to the dynamic hydrophobicity remains elusive.

Studies of the dynamic hydrophobicity of solid surfaces have been limited in the materials science field have been limited by the following factors: (i) Droplet shapes change gradually during sliding. Therefore, continuous and simultaneous measurement of both the sliding acceleration and the shape deformation are necessary for the comprehensive evaluation of the sliding behavior of liquid droplets. Appropriate systems to meet this objective were not developed until recently. (ii) Investigations of the sliding behavior of liquid droplets on the solid surface are mainly conducted in the field of fluid mechanics [57-59]; collaborative studies with materials scientists or surface chemists are scarce.

Analyses or modeling of the fluid mechanics of droplet sliding are often conducted with the assumption of constant sliding velocity. However, liquid droplets commonly show sliding acceleration in the early stage of sliding on hydrophobic surfaces with excellent watershedding properties. Measurement of the sliding behavior is generally feasible only for a limited range of samples. Therefore, corresponding research between sliding behavior and solid surface characteristics has not been well conducted.

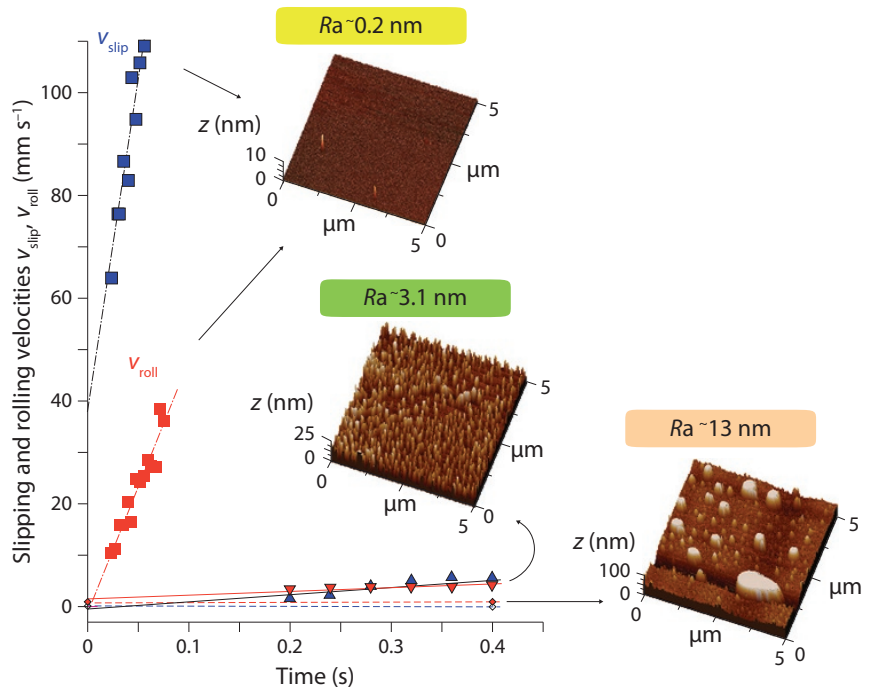

Figure 4. Effect of nanolevel heterogeneity on the sliding acceleration and rolling/ slipping ratio of water droplets on three hydrophobic fluoroalkylsilane coatings. All three coatings were prepared from fluoroalkylsilane (FAS17) by changing processing conditions, and possess three different roughness orders ( $R a)$. Insets are atomic force micrographs of the surfaces. $v_{\text {slipr }}$ slipping velocity; $v_{\text {roll, }}$ rolling velocity; squares, $R a=0.2 \mathrm{~nm}$; triangles $R a=3.1 \mathrm{~nm}$; dashed lines, $R a=13 \mathrm{~nm}$. A water droplet slides by caterpillar-like rotation flow with or without slippage on the solid-liquid interface. Absolute values of the sliding acceleration (slope of the line) and their ratio between rolling and slipping depend on nanoscale heterogeneity. Adapted from Ref. 56 (๔ 2009 Chemical Society of Japan).

Recently, studies of dynamic wettability have been stimulated by the availability of high-speed photography, the advancement of motionimage sequence analysis, and increasingly rapid computation. These technologies facilitate the advancement of studies not only of sliding behavior but also of other droplet behaviors such as horizontal motion, bouncing, evaporation and freezing. Moreover, the advance of nanoscale materials characterization has enabled studies of surface characteristics that govern the dynamic hydrophobicity of solids from the perspective of nanomaterials science.

Suzuki et al. [56] prepared fluoroalkylsilane coatings with different physical and chemical heterogeneities on silicon substrates by changing the processing conditions. They investigated the relationships among contact angle, sliding angle and sliding acceleration. Their coatings possess three different physical roughness orders ( $R$ a of $0.1,3.1$ and $13 \mathrm{~nm}$ ) and chemical heterogeneity (hydrophilic parts, probably because of some residual $\mathrm{SiOH}$ groups left by self-condensation or micelle formation), realized in the same material. Their research revealed that sliding acceleration and its behavior (rolling/slipping ratio during sliding, described later) are affected to a remarkable degree by nanoscale structural and chemical heterogeneity (Figure 4). This result implies that high smoothness and homogeneity are advantageous for obtaining surfaces with excellent dynamic hydrophobicity. In general, more precise process control is necessary for fluoroalkylsilanes than for alkylsilanes (e.g. octadecyltrimethoxysilane) to obtain highly smooth and homogeneous coatings. Perhaps this is the reason for the common trend of a large sliding angle in spite of the large contact angle on fluorocarbon surfaces as described above.

Yoshida et al. [51] prepared various smooth (Ra of ca. $0.3-0.5 \mathrm{~nm}$ ) fluoromethacrylate-methylmethacrylate (FMA-MMA) polymer coatings, and investigated the contact angle, sliding angle and sliding acceleration. Both the contact angle and the sliding acceleration on the coatings increased concomitantly with the number of fluorocarbons in the FMA. However, the contact angle hysteresis shows a different trend (Figure 5). In the longer and shorter fluoroalkyl chains (FMA-F17, -F19 and -F3), the contact angle hysteresis decreases concomitantly with increasing FMA concentration (and hence increasing contact 


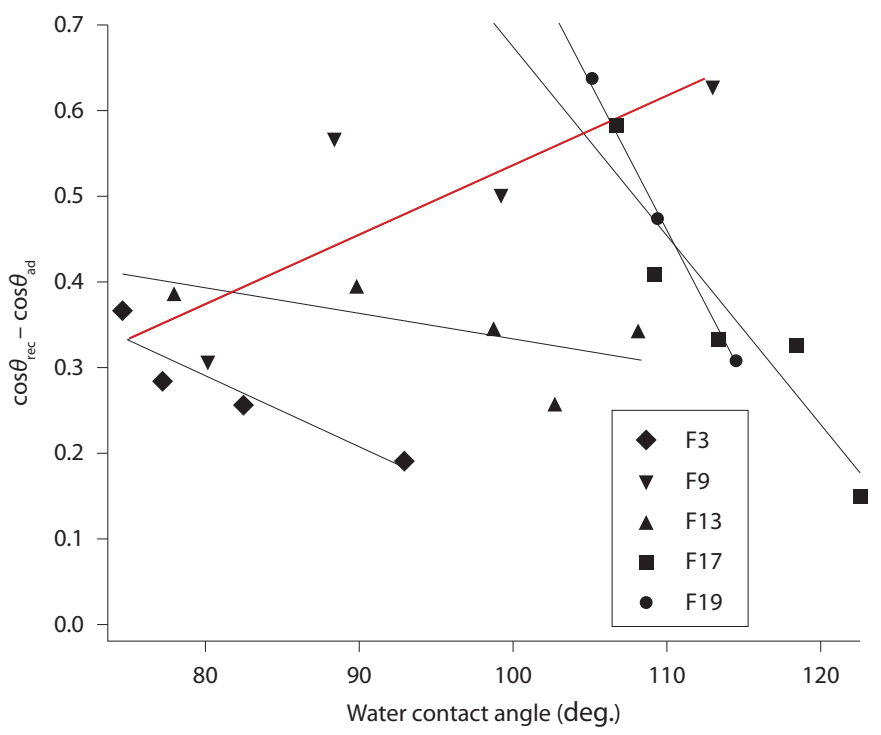

Figure 5. Effect of molecular length of FMA in FMA-MMA copolymer coatings on the contact angles and sliding angles of water droplets. The FMAs used were 2,2,2-trifluoroethyl methacrylate (FMA-F3), 2-(perfluorobutyl) ethyl methacrylate (FMA-F9), 2-(perfluorohexyl)ethyl methacrylate (FMA-F13), 2-(perfluorooctyl)ethyl methacrylate (FMA-F17) and 2-(perfluoro-7-methyloctyl)ethyl methacrylate (FMAF19). Although the contact angle hysteresis $\left(\cos \theta_{R}-\cos \theta_{A}\right)$ for the copolymers with long and short FMA chain (such as FMA-F3-MMA and FMA-F17-MMA) decrease concomitantly with increasing contact angle (i.e. with increasing FMA concentration in the copolymers), the FMA-F9-MMA copolymer showed a different trend. Adapted from Ref. 51 (๔ 2006 ACS).

angle), whereas it increases in the medium-length chain (FMA-F9). Honda et al. [60] attributed this result to the aggregation effect of the longer fluoroalkyl chain and the larger reorientation of the medium chain at the water-polymer interface. Reorientation of the short chain might be very slight because of its orientational freedom. The results suggest that the sliding angle and sliding acceleration are governed by different factors in these coatings.

Recent reports have described that slipping and rolling motions exist in the mode of sliding motion for a water droplet $[61,62]$. This result implies that the sliding acceleration depends on the sliding mode and that identification of the contribution of these two sliding modes to overall sliding acceleration or velocity is necessary for comparison of the water-shedding performance of hydrophobic solid surfaces. An effective approach for the direct observation of the internal fluidity of moving droplets is particle image velocimetry $[63,64]$. Using this technique, internal fluidity and velocity distribution during sliding are directly obtainable because of the use of tracking particles or their concentration dispersed in water (Figure 6). Studies using this system have revealed that the contribution of the slipping mode is large on a surface with excellent dynamic hydrophobicity, where the rolling/slipping ratio depends on nanoscale structural and chemical heterogeneities [56]. These factors affect the sliding acceleration strongly, just as they affect the sliding angle. However, the degree of influence of these factors has not been investigated systematically to date. Further detailed analyses are necessary.

It is noteworthy that the dynamic hydrophobicity described here is not determined by water-spreading kinetics, but by the water-shedding properties of the surface. The evaluation of dynamic hydrophobicity on a hydrophilic surface requires special care.

\section{Control of water droplets on solid surfaces}

The control of liquid droplets on solid surfaces has attracted much attention as an indispensable technology for use with various industrial items including microelectromechanical systems and lab-on-a-chip architectures, especially for hydrophobic coatings. Various investigations
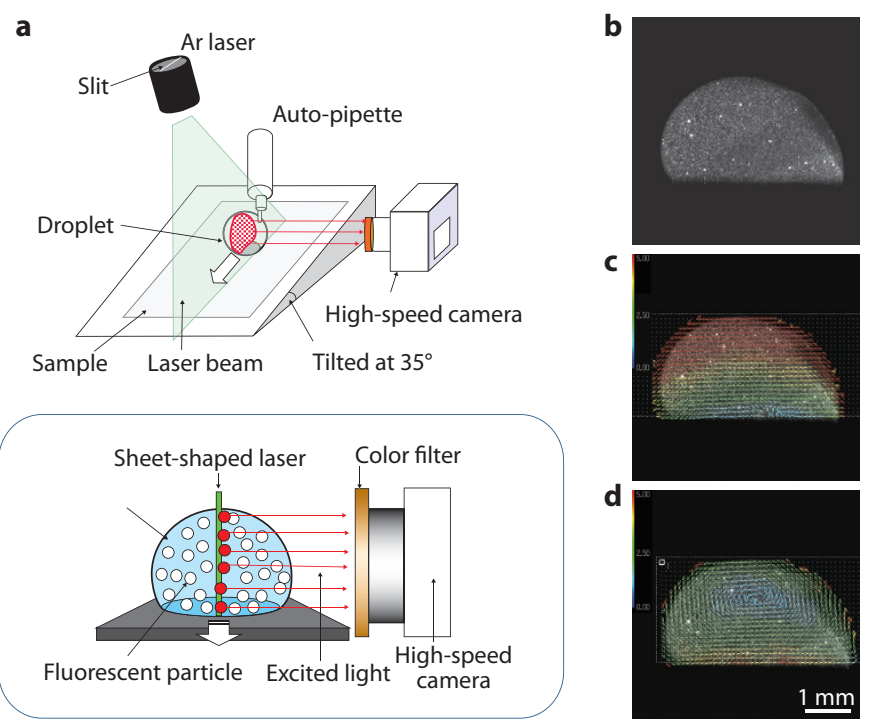

Figure 6. (a) Schematic illustrations of measurement of internal fluidity of water droplets during sliding using particle image velocimetry. Fluorescent particles are mixed with water. The practical viscosity and surface energy of the mixed liquid are almost identical to those of water. The particles emit light as a sheet-shaped laser irradiated perpendicular to the droplet. The image of the droplet sliding with light emission is recorded using a high-speed camera. The intensity and dispersion of the light in each pixel of the droplet image is tracked over a short time period and the velocity vector distribution in the image is calculated. (b) Practical image recorded using a high-speed camera. (c) Distribution of the velocity vector of the internal fluidity in the sliding droplet (velocity order, red $>$ yellow $>$ green $>$ blue). (d) Vertical velocity distribution of the internal fluidity in the sliding droplet after affine transformation, which adds an inverse velocity of the slipping component. The center of the rolling motion is visible in the droplet. Adapted from Ref. 64 (๑ 2007 AIP).

have been conducted to examine the motion of a liquid droplet on a solid surface using external fields such as gravity, heat gradients $[65,66]$, electric fields [67-81], magnetic fields [82], photo-illumination [83], blowing wind [84] and vibration [85].

Brzoska et al. [65] demonstrated the motions of water droplets induced by a thermal gradient and the resultant Marangoni effect on smooth hydrophobic silane surfaces. Pratap et al. [66] presented a lubricationtheory-based model for the thermocapillary motion of droplets on a solid surface, revealing that their model explains experimentally obtained results for decane drops moving on polydimethylsiloxane-coated glass.

A hydrophobic solid surface generally becomes hydrophilic upon application of an electric field $[67,68]$. Electrowetting is a microfluidic phenomenon that has grown explosively as a driving mechanism for various fluidic and electro-optic applications [69-73]. This approach involves modifying the surface energy of liquids on a solid surface using an electric field [74]. By designing appropriate electrode patterns or topographic structures on the substrate surface, variations in contact angle can give rise to changes in droplet shape, or in the motion of droplets [75-81]. Although electrowetting is useful in controlling droplets for droplet-based microfluidics, the high driving voltage often becomes a problem for device application [80]. Wang et al. [79] investigated microdroplet sliding on a polymer surface with and without an electric field using atomic force microscopy, and demonstrated that the presence of applied potentials increases the friction force between droplets and solid surface because of an increase in droplet diameter. Gunji et al. [81] demonstrated electric-field-driven self-propulsion of a water droplet on a solid surface using coplanar parallel-strip electrodes fabricated on a substrate and covered with a hydrophobic coating. The droplet motion is of a self-propelling nature; the water droplet leaves behind a moisture layer on the substrate surface, which shields the electric field on the droplet's trailing edge, thereby producing an imbalance in the Maxwell stress and produces a driving force. 


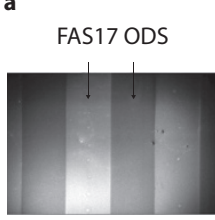

$500 / 500 \mu \mathrm{m}$

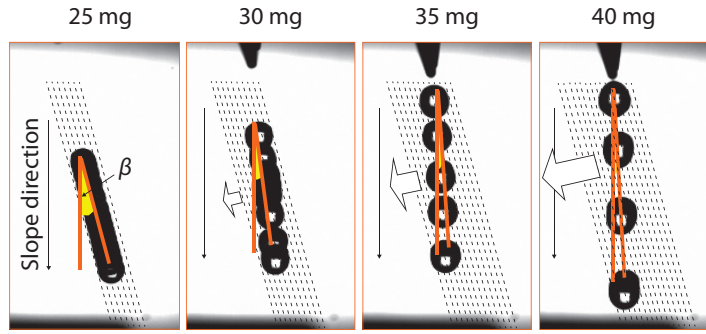

$10 \mathrm{~mm}$

Figure 7. (a) Field-emission scanning electron micrograph of the fluoroalkylsilane (FAS17) surface periodically line-patterned with octadecyltrimethoxysilane (ODS) at a line/space dimension of 500/500 $\mu \mathrm{m}$. (b) Evaluation of water droplet sliding behavior on these surfaces. (c) Sliding behavior of droplets with various masses on a $500 \mu \mathrm{m}$ line-surface rotated at $\Phi=13^{\circ}$ on a slope tilted at $35^{\circ}$. The image-recording interval is $200 \mathrm{~ms}$. The displacement angle $\beta$ depends on the droplet mass. CA, contact angle; WCA, water contact angle.

Cheng et al. [75] fabricated electrowettability on a dielectric device using a single-plate configuration with several steps for the control electrode array, and demonstrated that the threshold voltage needed to move the droplet $(2 \mu \mathrm{L})$ can be reduced to as low as $3 \mathrm{~V}$.

Hong et al. [82] demonstrated the no-loss reversible transport of microliters of a superparamagnetic liquid on a superhydrophobic surface using alternating magnetic fields. Ichimura et al. [83] achieved macroscopic motion of liquids on a flat solid surface by photoirradiation of a surface monolayer. In their research, the photoisomerization of surface azobenzenes was found to increase contact angle hysteresis and consequently produce directional motion. Hashimoto et al. [84] investigated the relationship between airflow and the motion of a water droplet on an inclined hydrophobic coating using a high-speed camera system and an open-circuit-type wind tunnel. They reported that the motion of a water droplet in an air stream can be classifiable into three types: sliding, stopping and climbing. They also determined that the wind velocity dependence differs between sliding and climbing acceleration. Dong et al. [85] identified numerical resonance modes for the vibration of a sessile drop on a hydrophobic surface. They reported that gravity enhances the first resonant mode and weakens the second mode, even though the positions of the resonance peaks do not differ substantially from those observed to have horizontal vibrations. When a drop is vibrated on a horizontal surface with asymmetric vibration, different modes are amplified asymmetrically during the forward and reverse strokes of a vibration cycle.

Chaudury et al. [86] demonstrated that a water droplet can be moved uphill merely by providing a spatial gradient in decyltrichlorosilane. Using that method, a liquid droplet is controllable not only in an external field but by the introduction of a gradient in solid surface characteristics such as shape [87], roughness [88,89] and surface energy [86,90], or a mixture of these [91]. Zhang et al. [87] demonstrated self-running, both horizontally and uphill, of water droplets by patterning a shape-gradient hydrophilic material in a hydrophobic matrix. The proposed driving force for the self-running of the water droplets in the system involves the difference in wettability of the surface materials, the low contact angle hysteresis of the surface materials and the space limitation of the shape-gradient transportation area. Fang et al. [89] demonstrated transportation of a single droplet using hydrophobicity differences on various textured surfaces. A droplet

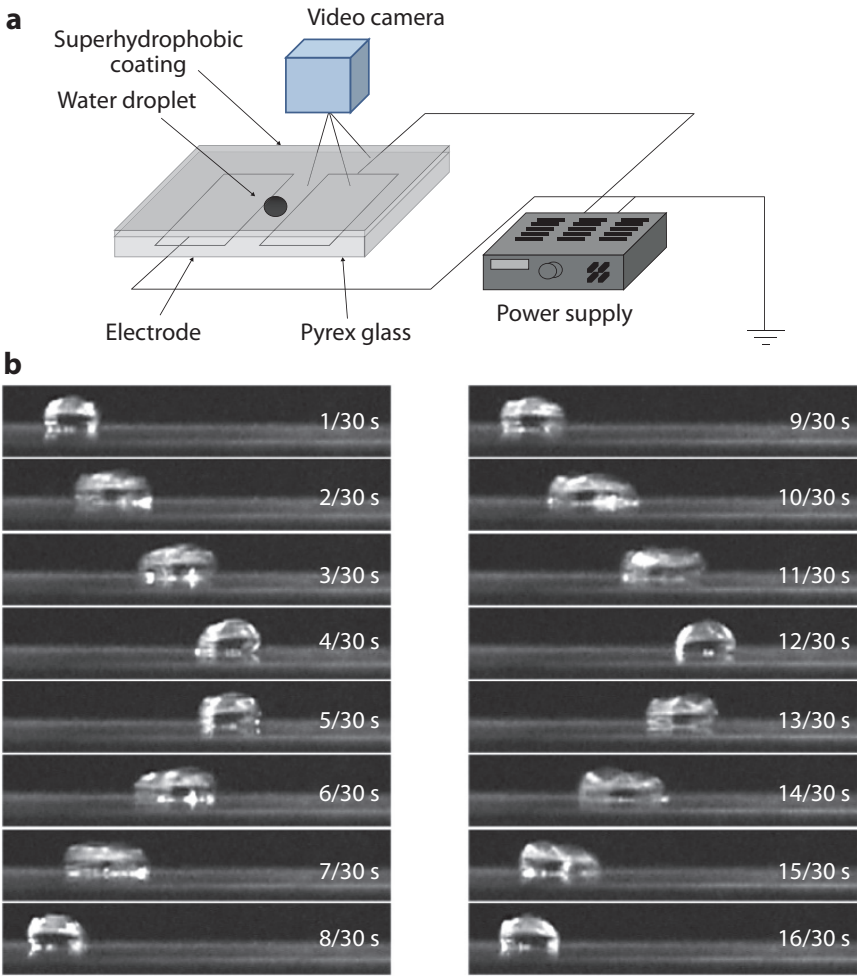

Figure 8. (a) Schematic illustration of the experiment system. (b) Sequence of microphotographs of the motion of a $20 \mathrm{mg}$ water droplet induced by the application of a $2.0 \mathrm{kV}$ alternating current $(3.7 \mathrm{~Hz})$ on a superhydrophobic film. The movement was induced up to a synchronizing frequency of $10 \mathrm{~Hz}$. Adapted from Ref. 80 (๔ $2002 \mathrm{JJAP}$ ).

on a surface that is patterned with a sequence of gradually increasing textured roughness regions can move continuously along the ascent of the surface roughness. Ito et al. [90] prepared a surface energy gradient using a moving mask and photolithography technique. They demonstrated droplet motion on the surface and reported that the water drop movement was regulated by the degree of gradation. In these surfaces, a Laplacian pressure difference is generated between advancing and receding sides of the liquid; the liquid droplets move spontaneously.

Micropatterned surfaces with different wettability are well known to provide wetting anisotropy [92]. The degree of that anisotropy depends on the line structure (line width), chemical composition, and its homogeneity. Using wetting anisotropy, the transportation of water droplets in the intended direction is feasible. Suzuki et al. [93] prepared a line-patterned surface using two silane coupling agents, then demonstrated that the selection of droplets is feasible by setting the surface rotating in-plane on the slope surface (Figure 7). This result is explained by the balance between gravitational and retentive forces. Directional transportation of a water droplet on a superhydrophobic surface with a hydrophilic path line was also demonstrated experimentally by Xu et al. [94]. Similar sliding anisotropy has been obtainable by preparing a line-patterned groove structure on a hydrophobic surface [95].

Droplet motion on a normal hydrophobic surface is not always fast, which sometimes constitutes a barrier to the application of surface wettability in industrial devices. A superhydrophobic surface, on the other hand, enables rapid transportation of water droplets. Various systems have been proposed to combine superhydrophobicity with external fields (Figure 8). Takeda et al. [80] controlled the motion of a water droplet on a superhydrophobic surface using an electric field. In their system, the Coulomb force of the electric charge provided to the water from the solid surface was the dominant driving force for water droplet movement. Recently, the internal fluidity of water droplets during transportation on superhydrophobic surface by an external electric field has also been analyzed using a particle image 
velocimetry system, revealing that the droplet undergoes slipping motion and that dielectrophoretic force plays an important role in movement by an electric field [96].

\section{Summary and prospects}

Studies of hydrophobic surface have to date mainly examined the relationship between the structure or chemical composition of a solid surface and the contact angle or sliding angle of water droplets. These are fundamentally examples of static hydrophobicity. For that reason, such studies provide no information related to dynamic hydrophobicity. Detailed investigations of the effects of solid composition, surface structure, their alignment, and their homogeneity on dynamic hydrophobicity are necessary for the design of surfaces with excellent watershedding properties.

Previously, the behaviors of liquids were treated mainly on the basis of fluid mechanics and the physical chemistry of the solid-liquid interface, and were investigated mainly from the point of view of surface science or colloid chemistry. The processing and properties of solid materials, however, are handled mainly in the field of materials science. To achieve a better understanding of the relationship between solid surface characteristics and dynamic hydrophobicity, interdisciplinary studies in these three academic fields are necessary. In addition to discussions from the surface chemistry or materials science perspectives, descriptions and discussions from the viewpoint of fluid mechanics and internal fluidity are indispensable. Collaborative studies for dynamic hydrophobicity might establish a new academic field situated on the border separating these three sciences.

Liquids and gels possess greater deformation flexibility than solids. The control of liquids is a key technology for new functional surfaces and devices, and such technology will also contribute to the reduction of greenhouse gas emissions through a reduction in friction drag leading to higher energy conservation. Basic research related to the control of liquid droplets on solid materials remains insufficient. As this is a vital technology for our future, further investigations are anticipated in this field.

\section{References}

1. L. A. Girifalco, R. J. Good, J. Phys. Chem. 61, 904 (1957).

2. T. Nishino, M. Meguro, K. Nakamae, M. Matsushita, Y. Ueda, Langmuir 15, 4321 (1999).

3. $\quad$ R. N. Wenzel, Ind. Eng Chem. 28, 988 (1936).

4. A. B. D. Cassie, S. Baxter, Trans. Faraday Soc. 40, 546 (1944).

5. R. E. Johnson Jr, R. H. Dettre, Adv. Chem. 43, 112 (1963).

6. M. Miwa, A. Nakajima, A. Fujishima, K. Hashimoto, T. Watanabe, Langmuir 16, 5754 (2000).

7. G. McHale, N. J. Shirtcliffe, M. I. Newton, Langmuir 20, 10146 (2004).

8. L. Gao, T. J. McCarthy, Langmuir 23, 3762 (2007).

9. Z. Yoshimitsu, A. Nakajima, T. Watanabe, K. Hashimoto, Langmuir 18, 5818 (2002)

10. M. Sasaki, N. Kieda, K. Katayama, K. Takeda, A. Nakajima, J. Mater. Sci. 39, 3717 (2004)

11. J. Drelich, J. D. Miller, A. Kumar, G. M. Whitesides, Colloid Surf. A 93, 1 (1994)

12. J. T. Woodward, H. Gwin, D. K. Schwartz, Langmuir 16, 2957 (2000).

13. N. A. Patankar, Langmuir 19, 1249 (2003).

14. A. Marmur, Langmuir 19, 8343 (2003).

15. J. Long, M. N. Hyder, R. Y. M. Huang, P. Chen, Adv. Coll. Int. Sci. 118, 173 (2005).

16. M. Nosonovsky, Langmuir 23, 9919 (2007).

17. A. Marmur, E. Bittoun, Langmuir 25, 1277 (2009).

18. A. Nakajima, K. Hashimoto, T. Watanabe, Monatsh. Chem. 132, 31 (2001).

19. X. Feng, L. Jiang, Adv. Mater. 18, 3063 (2006).

20. R. Blossey, Nature Mater. 2, 301 (2003).

21. M. Ma, R. M. Hill, Curr. Opin. Colloid In. 11, 193 (2006).

22. X. Feng, J. Zhai, L. Jiang, Angew. Chem. Int. Edit. 44, 5115 (2005).

23. M. Li et al., J. Phys. Chem. B 107, 9954 (2003).

24. A. Lafuma, D. Quéré, Nature Mater. 2, 457 (2003).

25. M. Nosonovsky, B. Bhushan, Nano Lett. 7, 2633 (2007).

26. K. A. Wier, T. J. McCarthy, Langmuir 22, 2433 (2006).

27. C. Dorrer, J. Rühe, Langmuir 23, 3820 (2007).
Y. Ito et al., Langmuir 23, 1845 (2007).

91. A. Shastry, M. J. Case, K. F. Böhringer, Langmuir 22, 6161 (2006).

92. M. Morita, T. Koga, H. Otsuka, A. Takahara, Langmuir 21, 911 (2005).

93. S. Suzuki et al., Appl. Surf. Sci. 254, 1797 (2008).

94. Q. F. Xu, J. N. Wang, I. H. Smith, K. D. Sanderson, Appl. Phys. Lett. 93, 233112 (2008).

95. A. D. Sommers, A. M. Jacobi, J. Micromech. Microeng. 16, 1571 (2006).

96. M. Sakai et al., Langmuir 26, 1493 (2010). 


\section{Author profile}

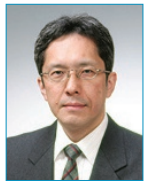

\section{Akira Nakajima}

Akira Nakajima earned his BE and ME degrees in inorganic materials science at the Tokyo Institute of Technology, Japan, in 1985 and 1987. He then joined Japan Energy Corporation as a research scientist. In 1997, he earned his PhD at Pennsylvania State University in the USA. He moved to the Research Center for Advanced Science and Technology at the University of Tokyo, Japan, in 1998 where he began research on hydrophobic coatings. He has established a venture company, Advanced Systems of Technology Incubation (ASTI) and became the company's chief technical officer in 2001. In 2003, he joined the faculty staff of the Tokyo Institute of Technology, where he currently holds the position of professor in the Department of Metallurgy and Ceramic Science. Between 2003 and 2007, he led the Super Hydrophilic/phobic Surface Project at the Kanagawa Academy of Science and Technology, Japan. 